Retraction

\title{
Retracted: Proanthocyanidins Antagonize Arsenic-Induced Oxidative Damage and Promote Arsenic Methylation through Activation of the Nrf2 Signaling Pathway
}

\author{
Oxidative Medicine and Cellular Longevity \\ Received 4 July 2020; Accepted 14 July 2020; Published 22 January 2021 \\ Copyright (C) 2021 Oxidative Medicine and Cellular Longevity. This is an open access article distributed under the Creative \\ Commons Attribution License, which permits unrestricted use, distribution, and reproduction in any medium, provided the \\ original work is properly cited.
}

Oxidative Medicine and Cellular Longevity has retracted the article titled "Proanthocyanidins Antagonize ArsenicInduced Oxidative Damage and Promote Arsenic Methylation through Activation of the Nrf2 Signaling Pathway" [1]. As raised on PubPeer [2], the article was found to contain images with signs of duplication in Figure 2, specifically the lower left of the flow cytometry shown in panels 2(a)1, 2(a)2, 2(b)3, and 2(b)4.

We asked the authors to provide the original uncropped and unadjusted images used to create all the figures, and all the raw data, i.e. all the underlying measurements for the statistics and averages shown in the graphs and tables. The authors did not provide the original images and raw data. However, they provided a corrected figure, which is available as Supplementary Materials. We consulted our Editorial Board, who recommended retraction.

\section{Supplementary Materials}

Figure duplication in Figure 2 of OMCL/8549035. (Supplementary Materials.docx). Corrected figure files (Supplementary Materials.rar). (Supplementary Materials)

\section{References}

[1] M. Xu, Q. Niu, Y. Hu, G. Feng, H. Wang, and S. Li, "Proanthocyanidins Antagonize Arsenic-Induced Oxidative Damage and Promote Arsenic Methylation through Activation of the Nrf2 Signaling Pathway," Oxidative Medicine and Cellular Longevity, vol. 2019, Article ID 8549035, 19 pages, 2019.
[2] "Proanthocyanidins Antagonize Arsenic-Induced Oxidative Damage and Promote Arsenic Methylation through Activation of the Nrf2 Signaling Pathway, PubPeer," June 2019, https:// pubpeer.com/publications/F36945569F53345B9852 F46240240B. 